\title{
21 A culture of proving in school mathematics?
}

\author{
Celia Hoyles \\ Institute of Education \\ University of London \\ United Kingdom
}

\begin{abstract}
It is commonplace in mathematics to present proving in a hierarchy of levels in which the empirical precedes the deductive. This paper questions the assumption that this is a matter of development of the latter from the former and presents an alternative sequence where the seeds of proving are sown in a computer-based construction process which requires an explicit description of relevant properties and relationships.
\end{abstract}

Curriculum policies, geometry, tools, proof.

\section{INTRODUCTION}

It is commonplace in mathematics to present proving in a hierarchy of levels in which the empirical precedes the deductive. Clearly students need to be able to distinguish empirical verification and pragmatic proof from deductive and conceptual proof (Balacheff, 1988) but the question remains as to how best might this be done. Is it a matter of development of the latter from the former or can links be forged between the two at every stage of schooling and throughout mathematical activity?

Our rationale ${ }^{1}$ is that we need to design new learning contexts which require the use of clearly formulated statements and definitions and agreed procedures of deduction but which also allow opportunities for their connection with empirical justification and the conviction this engenders. Previous work points to what might be the constituent components of such contexts: ease of transition between an enactive or visual form of proof and a sequence of deductions (Tall, 1995); 
emphasis on explanatory proofs which "illustrate as well as dispel doubt" (Hanna, 1995); use of generic proofs where work is done on a particular example chosen so as to be "typical of the whole class of examples and hence the proof is generalisable" (Tall, 1979). Yet in order for such situations to be effective in the classroom, the first step must be to tailor them so as to fit into a specific curriculum context-in this case that of the UK National Curriculum.

\section{THE NATIONAL CURRICULUM IN ENGLAND AND WALES}

In UK schools, considerable attention has been paid to the process of proving (see, Mason et al., 1982) and a sequenced process approach is now set out in Attainment Target 1 (AT1) of the National Curriculum, titled Using and Applying Mathematics. This places emphasis on investigating and conjecturing with empirical checks rather to the neglect of justification within any sort of deductive framework. Yet, despite these changes, the picture of students' appreciation of the nature of proof remains disappointing. Some recent research by Coe \& Ruthven (1994) into the proof practices of students who have followed this curriculum, suggests, rather unexpectedly, that nothing appears to have changed and students remain locked in a world of empirical validation. Even more surprisingly, given the emphasis on process in the curriculum reforms, the researchers also report that students show little attempt to explain why rules or patterns occur, or to locate them within a wider mathematical system. Clearly, the shift to a process-oriented perspective is an understandable attempt to move away from the meaningless routines that characterised what was largely geometrical proof in an earlier period. But in trying to remedy one problem, others may have emerged and there is a pervasive belief, amongst influential groups in the UK, that students' understanding of the notion of proving and proof in mathematics has deteriorated (see, for example, London Mathematical Society, 1995).

This argument is difficult to sustain in the absence of systematic evidence. Having followed the new curriculum, what do students judge to be the nature of mathematical proof? What do they see as its purposes? Do they see proving as verifying cases or as convincing and explaining? Do they forge connections between the different functions of proof or do these functions remain fragmented and isolated from each other? What are their teachers' views? Although we have a National Curriculum, are there variations in how it is delivered and experienced and if so why and what are the implications for student learning?

Our ongoing research ${ }^{2}$ has gone some way to answer these questions. Its results from a nation-wide survey (Hoyles, 1997) confirm some problems, yet point to where and how improvements might be made. Even in the highlyselected group of high-attaining Year 10 students (age about 15 years) responding to our survey throughout England and Wales, the majority use narrative or 
empirical rather than formal argument, in line with previous research where students followed a more traditional curriculum.

But our results also suggest that despite their lack of experience with formal proof, the students have developed considerable expertise in conjecturing, arguing and explaining in 'everyday' language. Additionally, in contrast to previous studies, we have found that most students are aware of the limitations of empirically-rooted arguments and recognise that a general, analytic justification is required: they simply do not know how to construct it. These gains must not be sacrificed in the pursuit of injecting more rigour and suggest that there is a need to introduce more formal mathematical justification, provided that care is taken to connect it with informal argumentation.

\section{INTRODUCING THE COMPUTER}

Our assumption is that 'the computer' can offer just such a new context and can help to build bridges between the empirical and the deductive. This assertion must be treated with caution-we cannot assume that the introduction of the computer will bring about change-at least, not change for the better. To date, work with computers in mathematics education has largely been concerned with construction and the potential of software to aid the transition from particular to general cases-specific instances can be easily varied by direct manipulation or text-based commands and the results 'seen' on the computer screen (see, for example, Laborde and Laborde, 1995). Yet, even if students develop a sense of how certain 'inputs' lead to certain results, there remains the question of how to develop a 'need for proof' and to point to a way to explain or even to prove as part of, rather than added on to, this constructive process. Unless we address this issue, there is a danger of limiting the mathematical work of the majority to even more convincing empirical argument-for example, using powerful dynamic geometry tools simply to measure, spot patterns, generate cases, and provide data.

There is an alternative which we are in the process of investigating which sets out through computer interaction to motivate an explicit characterisation of a mathematical activity - its mathematical heart (or hearts)by which students have to attend to the relationships they are setting up and which provides a rationale for their necessity. The seeds of learning to prove can therefore be sown in a construction process which requires an explicit description of properties and relationships-the 'givens' at the start of a proof or the mathematical model of the situation. The key insight is that parts of this model are built into the fabric of the computer medium - the tools we choose to provide- thus shaping the types of actions that are possible: they do not only exist in the mind of the learner. The level of what can be thought about, talked about, is notched up a rung or two and abstractions made which are situated within the existing model, expressed through 'statements' (mouse clicks, pieces of programs, etc.) which are already expressions of mathematical abstractions (for an 
elaboration of these theoretical ideas, see Noss and Hoyles, 1996). These objects and relationships can be acted on, visualised, and, although they might appear to be concrete and particular, they embed the seeds of a general case and a deductive framework.

Thus the scenario we envisage is one where students construct the objects for themselves on the computer, conjecture about the relationships between them, and check the truth of their conjecture with the tools available. This hopefully leads them to be able to justify their conjectures by reflection on their constructions and the computer feedback. This sequence is not undertaken in a linear fashion but one which is spiral and iterative ${ }^{3}$. It is in this way, we suggest that constructing and proving can be brought together in ways simply not possible without an appropriate technology.

\section{PEDAGOGICAL APPROACH}

Clearly the activities around the computer and the way a teacher fosters movement to and fro from formal to informal, rigorous to intuitive, are both crucial. Formal proof then becomes simply one facet of a proving culture, revitalised by the 'experimental realism' of the computer work (Balacheff and Kaput, 1996).

We have devised activities in both algebra and geometry which try to conform to these criteria. Our activities were developed after analysing students' responses to the paper and pencil survey and reviewing school textbooks and examination papers. The activities in each domain were designed for 15-year-old students of above average mathematical attainment and were planned to span three sessions supplemented with homework. The sessions comprise computer-integrated activities with students working in pairs and whole group discussion. In the following sections, I provide a brief description of some of the geometry activities which incorporate the use of a dynamic geometry system, Cabri Geometry. Following this section, I report some student and teacher responses.

\section{SOME ILLUSTRATIVE GEOMETRY ACTIVITIES}

In the first of the three geometry sessions, we try to foster the notion that proof must be general. Students become familiar with a range of Cabri tools by experimenting with the standard construction items (parallel lines, perpendicular lines, symmetrical points, midpoints etc.) as well as with the new tools, Compass and Angle Carry, which we have added to the menu ${ }^{4}$. In the activities themselves, the students are free to choose any computer tools that they feel would be useful-and clearly their choices shape their conjectures and subsequent problem solving strategies.

The basis of the first activity is an exploration of the conditions of congruency of triangles. Students in the UK curriculum are expected to 'understand the 
congruence of simple shapes', 'be able to recognise congruent shapes at Level 4' (average performance of 11 year old children) but are only expected to use these conclusions for solving problems if they are of 'exceptional performance'. Similarly, in ATI, it is not until students reach the heights of exceptional performance that they are expected to use mathematical language and symbols effectively in presenting a convincing reasoned argument. So we expected our tasks to be challenging.

Once the students are comfortable with this small set of Cabri tools, they are introduced, as a group, to the topic of congruent triangles: figures with corresponding angles and line segments of exactly the same size. The triangle shown in Figure 1 is on the computer for each student pair, with the measures of all side lengths and angles marked.

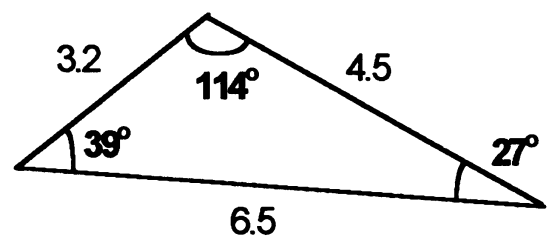

Figure 1 The original triangle.

The following question is then posed:

"How much information is required to construct a triangle that is congruent to the one given? Find as many minimum sets as you can."

A poster, as illustrated in Table 1 overleaf, showing several combinations of the given information (e.g., SIDE-ANGLE-SIDE, SIDE-SIDE-SIDE) is pinned up on the wall and reproduced in A4 so each student pair can record their own work.

The students in pairs are asked to consider the condition SIDE-ANGLE-SIDE and test if these three constraints are sufficient to ensure congruence-i.e., that the triangle they construct will always be congruent to the original triangle even if the specific measures of the original triangle are changed. Thereafter, they can choose any of the other sets of conditions - to predict whether the information is sufficient to ensure congruency and record this on their sheet; verify and check their prediction on the computer by testing whether the construction under the given conditions "messes up" (see Healy, et al., 1994) after dragging points on the constructed triangle or on the original triangle. Finally, the students are encouraged to justify their conclusions about which sets of measures are sufficient to ensure congruency and which are not sufficient, exploiting the insights gained from the construction tasks and their checking procedures. 
174 Information and communications technologies in school mathematics

Will the information shown ensure congruence?

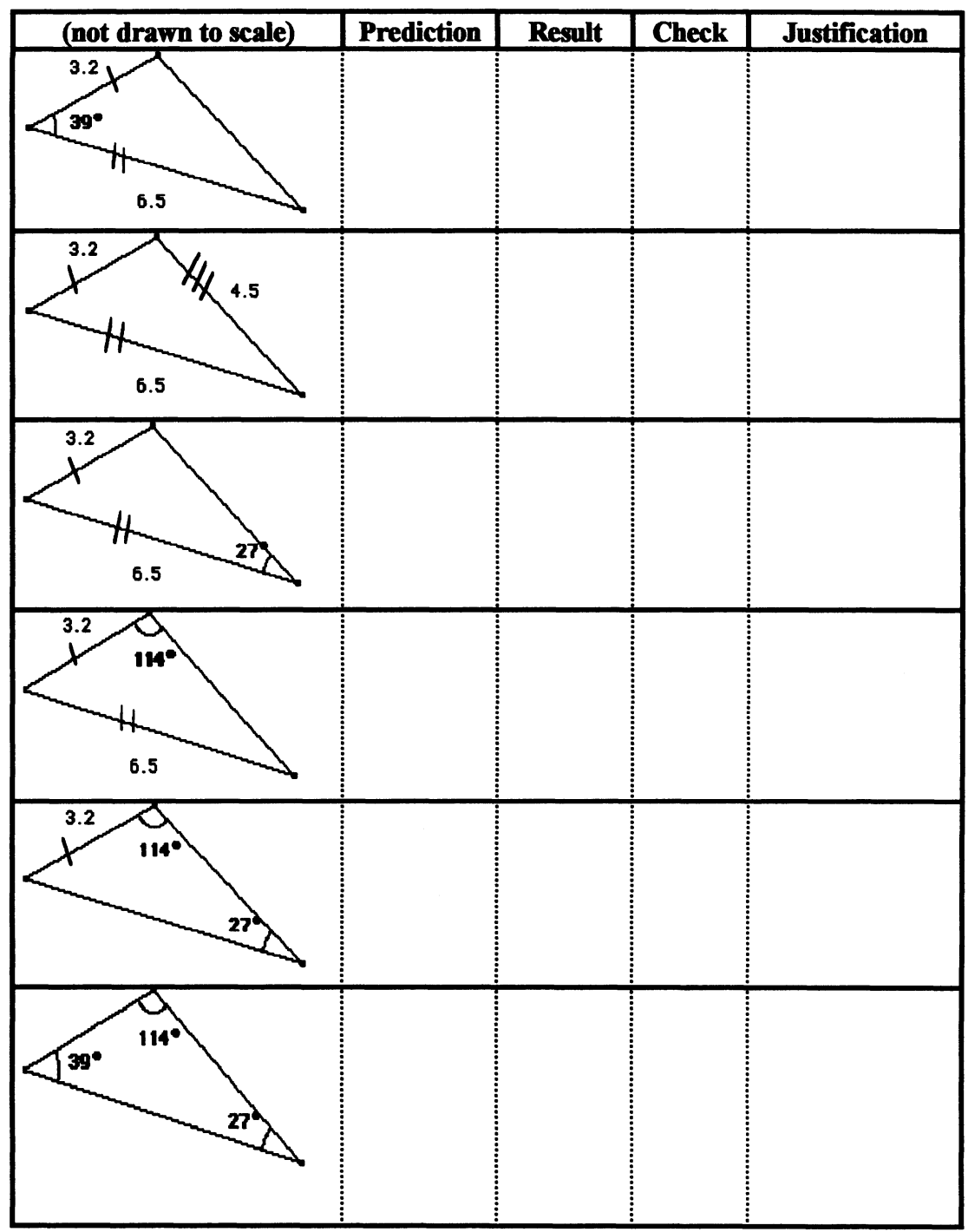

Table 1 Exploring congruent triangles. 
The session ends by drawing everyone together to compare results from the individual record sheets and coming to some group conclusions which are entered on the poster-sized table. The homework consolidates and allows reflection on the ideas met in the session using a standard textbook format. One example homework task is shown in Figure 2.

$\mathrm{AC}$ is a horizontal line; $\mathrm{BD}$ is a vertical line,

Use congruent triangles to explain why $\mathrm{AB}=\mathrm{BD}$

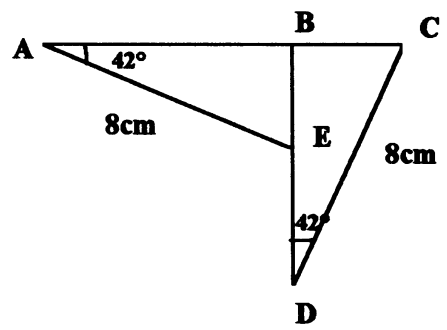

Figure 2 A homework exercise after the first session.

The second session sets out to support the writing of formal proofs by building on the actions, conjectures and explanations engendered earlier. Formal proof is introduced by discussing a selection of student proofs, carefully chosen from those written previously in answer to the homework questions. These include: an example where a condition that looks true visually but is not true in general is used in the proof; a circular argument where what has to be proved is used in the proof; and a narrative proof which is ambiguous. These examples are used to motivate the use of a precise formal language for proof, the careful separation of the givens from what has been proved and the need for a logical chain of deductions and reasons.

To introduce the construction activities for the session, a new poster is presented showing several quadrilaterals-parallelogram, rectangle, rhombus and square-where again, the students have their own copy to complete. The group as a whole agree the defining properties of these quadrilaterals, then, at the computers, they are shown the tool Check Property-how Cabri can check if something which looks true is generally true and if not can provide a counterexample. They then construct different quadrilaterals, each time checking if their constructed figure satisfies the negotiated list of defining properties using Check Property and finally trying to write a justification.

The final session aims to provoke students so they ask the questions, why? and how? for themselves. At the computers, the students are introduced to the Soft Locus and Angle Bisector tools before being presented with the following challenge:

Can you construct a quadrilateral in which the angle bisectors of two adjacent angles cross at right angles? What are its properties? 
This task is by no means trivial. All the students and most teachers who have tried it have no immediate intuition as to the characteristics of the required quadrilateral. They can only work experimentally to help them to 'see' its shape-after which they can try to prove it. Having completed this task, they are presented with what we anticipated would be a simple extension, a construction which is clearly impossible if account is taken of the construction just completed and the necessary relationships specified.

Can you construct a triangle with the same condition (i.e., with two adjacent angle bisectors perpendicular)?

We were interested to see if the students needed to construct the simpler figure, the triangle, or could they simply connect this triangle activity with the condition they had proved must apply for the quadrilateral.

\section{REFLECTIONS}

Here I will present a range of interesting observations based on our pilot work.

\section{Working Experimentally}

In the first session, to explore the congruence condition SAS most of the students worked experimentally, in fact testing all possible cases. They used compass to create two concurrent circles, set up two line segments joining the centre $\mathbf{O}$ to each of the circumferences, measured the angle between them and then moved the point on one of the circles, say $\mathrm{X}$, until they obtained the matching angle of $39^{\circ}$ (see figure 3).

They easily tested empirically that the only two triangles they could create were congruent to the original-by measuring all the sides and angles and by testing that the congruence held when dragging the original triangle. In fact, one is a reflection of the other. After all this work, they were also able to come up with very convincing explanations as to why the three measures were sufficient because basically they had tested all cases by moving their point on the inner circle to every possible position.

\section{Seeing the counter-example}

Using angle carry proved to be particularly useful when it came to test out the SIDE-SIDE-ANGLE set of conditions (the third in Table 1). Students could set up the two circles with radius 3.2 and 6.5 with compass and then carry the angle of $27^{\circ}$ to vertex $X$ on the outer circle. As can be seen in Figure 4, it is easy to spot the two points of intersection of this line with the inner circle and thus identify the counter-example-a triangle which satisfies the conditions, but is not congruent to the original. 


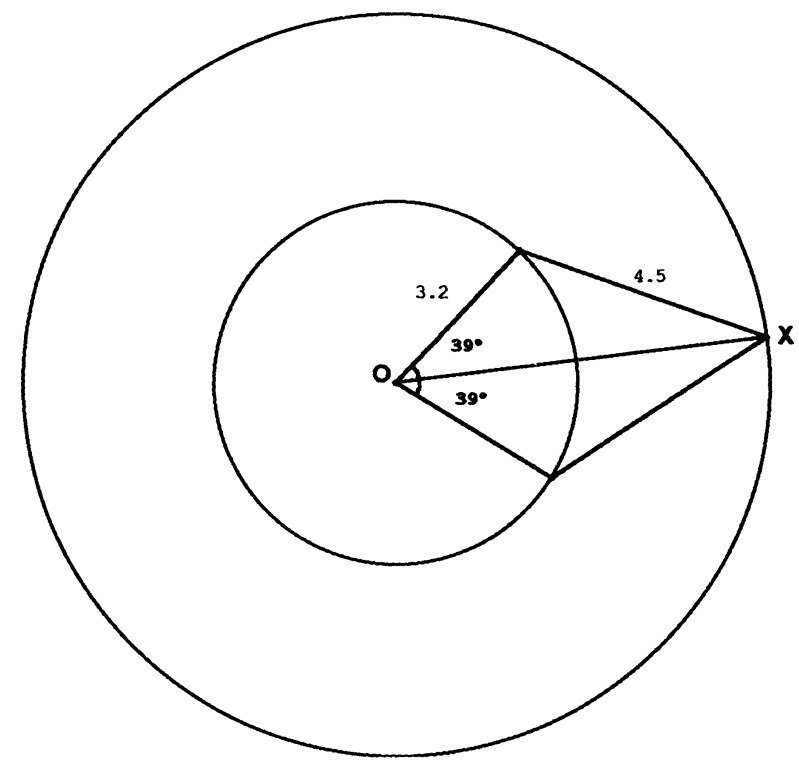

Figure 3 Testing SAS experimentally.

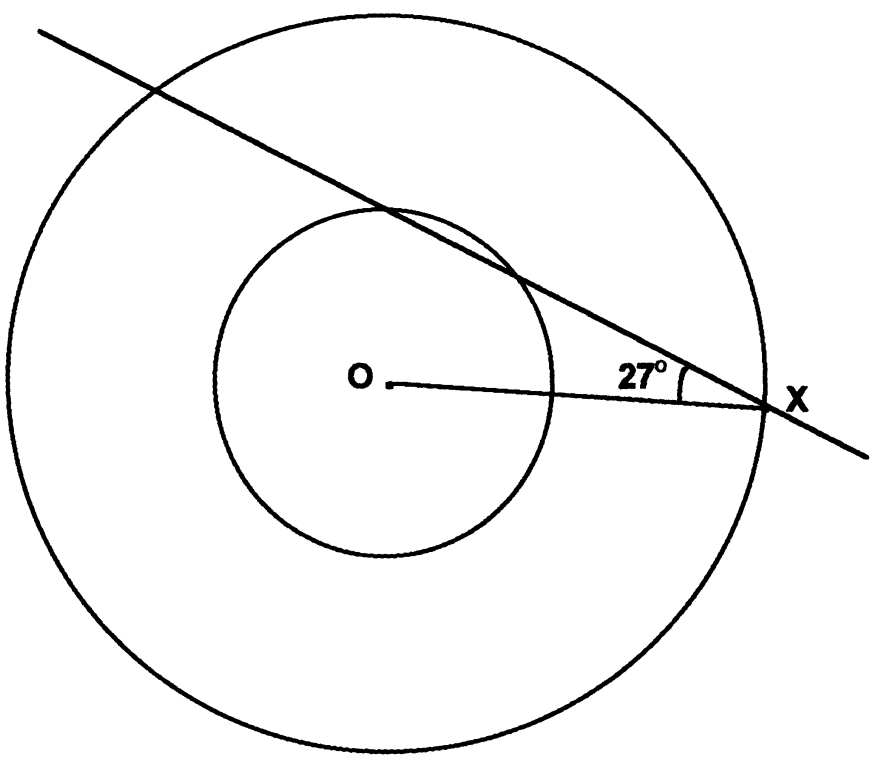

Figure 4 Finding a counter-example. 
As an addendum, it is illuminating to report how teachers have reacted to our activities. We have used these congruency tasks to great effect with teachersmost of whom have never thought about why the congruency constraints 'work' or indeed the exact conditions that are necessary. With teachers, we have gone further than described above. After setting up the situation illustrated, they can immediately see that they will only have one triangle-so congruence will be guaranteed-if the line from $X$ is a tangent to the inner circle. They can try to make this happen by manipulating the original triangle to make the line 'look' as if it is touching. Watching how the two triangles come together, trying to explain what is going on and much to their surprise reinventing the RHS $^{5}$ condition has caused considerable pleasure and insight.

All the teachers with whom we have worked have conjectured that the fourth set of conditions in Table 1 is not sufficient to guarantee congruency-that is SSA where the side opposite the given angle is the longest of the three ${ }^{6}$ Their experimental work on the computer soon convinced them that this was not true and indeed why not-again, by trying all possibilities but also by reflecting upon the intersection of the angle carry with the circles.

\section{Connecting construction and proof}

Turning to the last activity, again almost all the students worked experimentally: that is, set up the vertices for the quadrilateral, A, B, C, and D, constructed the angle bisectors at $A$ and $B$ and then moved say $D$ to try to satisfy the conditions of the problem. After finding one position of $D$ that made the bisectors perpendicular, they could explore other possibilities to help them to move from this specific solution to other solutions and thus to specify the characteristics of the general case. One way to do this to leave a trail of basic points at the 'successful' positions D as illustrated in Figure 5. Another way (usually following the first) is to use soft locus as shown in Figure 6.

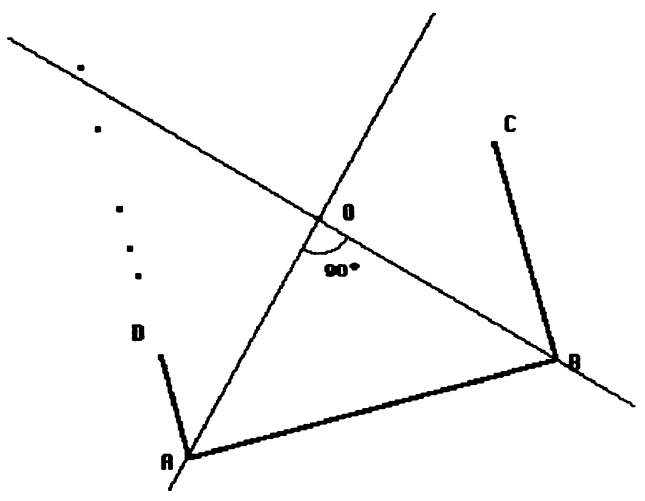

Figure 5 Perpendicular angle bisectors for a quadrilateral-plotting some points. 


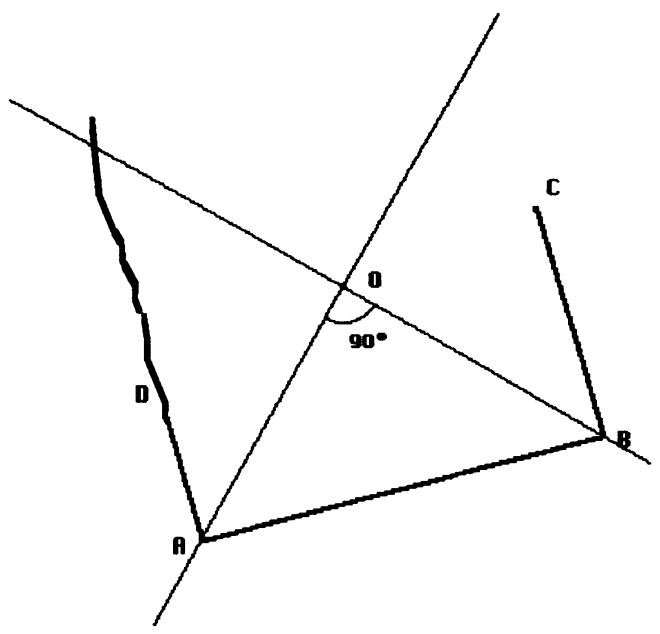

Figure 6 The soft locus.

We anticipated that these strategies would help the students see that the sides $\mathrm{AD}$ and $\mathrm{BC}$ must be parallel and once making this observation they would be able to construct the quadrilateral which satisfies the given conditions, reflect on all its properties and then prove that it must be a trapezium. All these steps, we surmise, were only remotely possible after the experimental work, with the visual object there in front of them.

\section{Disconnections}

But after all this struggle, what was even more fascinating to us was that the students did not see that the necessity for the parallel lines following the condition of perpendicularity of the angle bisectors - a necessity they had 'felt' and proved-meant that the construction of the triangle in the last challenge was impossible. They all began to try to construct it, only then realising that it could not be done. Then they quickly understood what was going on and laughed-“of course it is impossible!"

Why is there still this disconnection? Is it to do with ownership of the 'proofs' about quadrilaterals? Or the student views of proof? Or about the didactic contract which indicated somehow such a triangle existed? Or more generally does it relate to the continuing fragmentation of knowledge even in the face of all our efforts are towards making connections? Exactly the same phenomenon was noted in our parallel set of algebra activities and this is certainly something we will investigate further in the next few months. 


\section{CONCLUSION}

So what have students learned and what is new? Certainly, students made considerable progress as measured by their responses to our written proof questionnaire administered at the beginning and end of the sessions. They progressed in the way they wrote, explained and recognised proofs. We can also vouch for the enthusiasm of the students and teachers. There are no vestiges of the dull alienation of formal proofs and students positively enjoyed sorting out their givens, arguing, trying to explain and write their proofs. They enjoyed learning the game of proving and felt part of it.

Some commentators may question whether the presence of the computer was necessary to achieve even these limited ends. Without doubt it was - to make construction methods explicit, to allow reflection on properties, to check things out and obtain immediate feedback but most crucially to foster a conjecturing, experimental atmosphere that the teacher could exploit to introduce formal proof in ways which matched rather than supplemented student constructions. For example, proof by exhaustion (that SAS guaranteed congruency) is not possible without technology and, although we know it is not actually exhaustive; it is convincing and certainly helps the students to connect constructions to the proving process.

There are still surprises. For example, the girl who said after writing her formal proof "Don't we have to write all that stuff?" - surely a reflection of the influence of our curriculum where essay reports of processes are expected after 'doing investigations'. There is clearly food for thought in that not one student immediately recognised the impossibility of our final construction. But our success is that our students seemed to have glimpsed the power of the proving process, the aesthetic of a formal proof and have begun to connect it to their own constructive endeavours. We believe that with more such activities integrated throughout the curriculum, real and long term progress in this difficult area might be made.

\section{Notes}

1 The work reported in this paper was carried out with Lulu Healy. I wish to acknowledge her central contribution as well as the influence of our collaborations with Richard Noss.

2 Justifying and Proving in School Mathematics, Funded by the Economic and Social Research Council (Grant No. R000236178, Hoyles and Healy 1994).

3 The analysis undertaken in Noss and Hoyles 1996 underpins this key concern: to develop a more conscious appreciation of mathematical abstracting as a process which builds upon layers of intuitions and meanings rather than replaces them.

4 Compass enables students 'to carry' a length by modelling the use of a compass to produce a circular locus. 
5 RHS means right angle, hypotenuse, side.

6 I have not found a single text book in the UK that mentions this condition for congruency-although I understand that this set of measures is taught in other countries.

\section{REFERENCES}

Balacheff, N. (1988). Treatment of Refutations: Aspects of the Complexity of a Constructivist Approach to Mathematics Learning. In E. Von Glasersfeld (ed.), Radical Constructivism in Mathematics Education. Reidel.

Balacheff, N. and Kaput, J. (1996). Computer-Based Learning Environments in Mathematics. In A. Bishop, K. Clements, C. Keital, J. Kilpatrick and C. Laborde (eds.) International Handbook of Mathematics Education, Part 1. Dordrecht: Kluwer, 469—505.

Coe, R. and Ruthven, K. (1994). Proof Practices and Constructs of Advanced Mathematics Students. British Educational Research Journal, 20(1), 41-53.

Hanna, G. (1995) Challenges to the Importance of Proof. For the Learning of Mathematics, 15(3), $42-49$.

Healy, L., Hoyles, C., Hoelz, R. and Noss, R. (1994). Messing Up: Reflections on Introducing Cabri Géomètre. Micromath 10(1), 14-16.

Hoyles, C. (1997) The Curricular shaping of students' approaches to proof. For the Learning of Mathematics 17(1), 1-16.

Laborde, C. and Laborde, J. (1995). What About a Learning Environment Where Euclidean Concepts are Manipulated with a Mouse? In A. di Sessa, C. Hoyles, R. Noss, and L. Edwards (eds.) Computers for Exploratory Learning. Springer Verlag.

London Mathematical Society, Institute of Mathematics and its Applications, Royal Statistical Society (1995). Tackling the Mathematics Problem. London, U.K: London Mathematical Society.

Mason, J., Burton, L. and Stacey, K. (1982). Thinking Mathematically. London: Addison-Wesley.

Noss, R. and Hoyles, C. (1996). Windows on Mathematical Meanings: Learning Cultures and Computers. Dordrecht: Kluwer Academic Publishers.

Tall, D. (1995). Mathematical growth in elementary and advanced mathematical thinking. In L. Meira and D Carraher (eds.) Proceedings of the Nineteenth International Conference for the Psychology of Mathematics Education, 1 61-75. Recife, Brazil: Universidade Federal de Pernambuco. 
182 Information and communications technologies in school mathematics

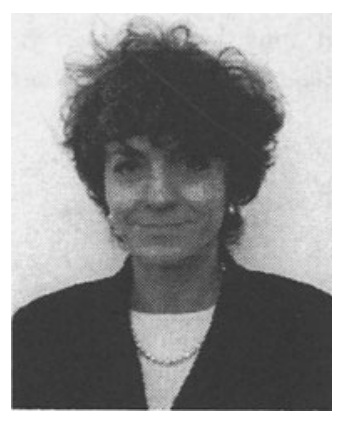

Celia Hoyles was appointed Professor of Mathematics Education at the Institute of Education, University of London in 1984 after her earlier career as a teacher in London schools and a polytechnic principal lecturer. She has an international reputation as a researcher and consultant in mathematical education, giving talks throughout the world and publishing some 200 articles for academics and teachers. Professor Hoyles has directed many research projects concerned with primary and secondary mathematics, particularly relating to the use of computers. Her recent research work on proof in secondary school mathematics has pointed to important strengths and weaknesses of the UK National Curriculum. 